\title{
Percepções e Sentimentos de Gestantes e Puérperas sobre a Pré-Eclâmpsia
}

\section{Perceptions of pregnant and postpartum women's feelings about preeclampsia}

Daniela Vasconcelos de Azevedo ${ }^{1}$, Ana Cristina Pinheiro Fernandes de Araújo ${ }^{2}$, Íris do Céu Clara Costa ${ }^{2}$ e Antônio Medeiros Júnior ${ }^{2}$

1 Universidade Estadual do Ceará (UECE), Fortaleza, Ceará, Brasil dvazevedo@yahoo.com.br 2 Universidade Federal do Rio Grande do Norte (UFRN). Natal, Brasil. crysaraujo@uol.com.br, iris_odontoufrn@yahoo.com.br, amjunior@ufrnet.br

Recebido em 18 Fevereiro 2009/Enviado para Modificação em 16 Possa 2009/Aprovado em 25 Possa 2009

\section{RESUMO}

Objetivo Compreender como gestantes e puérperas com pré-eclâmpsia, percebiam e vivenciavam a pré-eclâmpsia.

Material e métodos Entrevistas e obsenvação foram realizadas entre fevereiro e junho de 2007, no ambulatório e internamento de uma maternidade pública no Nordeste do Brasil, com 20 mulheres que apresentaram pré-eclâmpsia. A análise foi baseada na hermenêutica de Gadamer, com a construção de categorias temáticas.

Resultados As mulheres percebiam a gravidade e alguns riscos aos quais estavam expostas, porém conheciam pouco acerca da pré-eclâmpsia e suas conseqüências e pouca informação foi repassada pelos profissionais de saúde que as acompanhavam. O sentimento mais presente entre elas foi o medo de morrer, de perder o bebê e o medo por não conhecer a doença.

Conclusões A percepção da gravidade desta doença somada a pouca informação recebida durante o tratamento intensificou o medo destas mulheres. A adoção, por parte dos profissionais de saúde, de uma maior humanização do pré-natal de alto risco, em especial em relação à pré-eclâmpsia possibilitaria uma abordagem que considerasse a dimensão emocional das gestantes e puérperas durante as consultas.

Palavras-Chave: Pré-eclâmpsia, cuidado pré-natal, emoções (fonte: DeCS, BIREME).

\section{ABSTRACT}

Objective The present study was aimed at understanding how pregnant and postpartum women with preeclampsia perceived and experienced their condition. Materials and Methods Interviews and observation were conducted with 20 women having preeclampsia between February and June 2007 in the outpatients department and hospitalisation ward of a public maternity hospital in north-eastern Brazil. The analysis was based on Gadamer's hermeneutics, with construction of thematic categories.

Results The women were aware of the gravity and some of the risks to which they were exposed; however, they had little knowledge about preeclampsia and its consequences 
and little information was given by the health professionals who were treating them. The most present feeling among these women was fear of death, fear of miscarriage and fear caused by lack of information about the disease.

Conclusions The perception of the gravity of the disease coupled to the little information given during treatment increased fear amongst these women. Health professionals making high risk prenatal assistance more humanised (especially regarding preeclampsia) could facilitate an approach which would take the disease's emotional dimension into account during pregnant and postpartum women's clinical appointments.

Key Words: Preeclampsia, pregnancy, prenatal care, perception, emotions (source: $\mathrm{MeSH}, \mathrm{NLM}$ ).

\section{RESUMEN}

Percepciones y sentimientos de mujeres gestantes y puérperas sobre la preeclampsia

Objetivo Comprender como las embarazadas y puérperas perciben y viven la preeclampsia.

Material y métodos Se realizaron entrevistas y observaciones entre Febrero y Julio de 2007, en un hospital público de maternidad del Estado de Rio Grande do Norte, en el Noreste de Brasil, con 20 mujeres que presentaban preeclampsia. El análisis se basó en la hermenéutica de Gadamer, por medio de la construcción de categorías temáticas.

Resultados Las mujeres percibían la gravedad y algunos riesgos a los cuales estaban sometidas, pero conocían poco de la preeclampsia y sus consecuencias. Poca información les dieron los profesionales de salud que las trataban. Los sentimientos más presentes fueron el miedo a morir y perder sus niños, por no conocer la enfermedad.

Conclusión La percepción de la gravedad de la enfermedad y la poca información recibida durante el tratamiento, intensificó el miedo de las mujeres. La adopción de una mayor humanización por parte de los profesionales de la salud, con respecto a la atención pre-natal de alto riesgo, y en especial en relación con la preeclampsia, posibilitaría un enfoque que pueda considerar la dimensión emocional de las embarazadas y las mujeres que se encuentran en el periodo pos embarazo, en la consulta medica.

Palabras Claves: Preeclampsia, atención pre-natal, emociones (fuente: DeCS, BIREME)

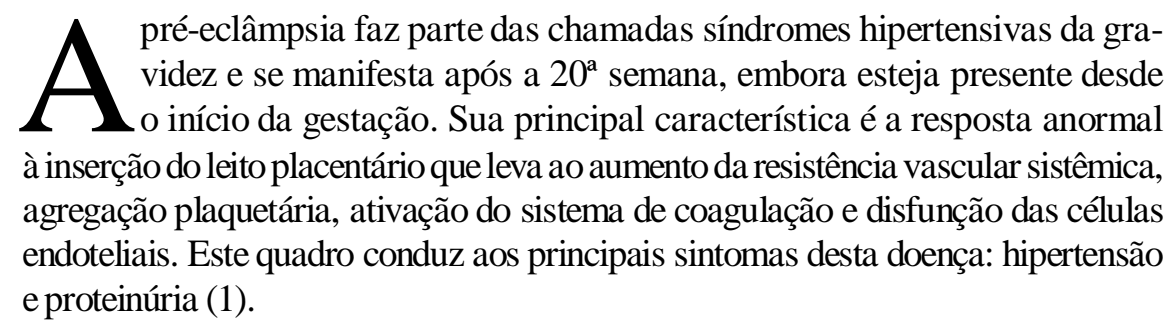


As causas do surgimento da pré-eclâmpsia ainda permanecem desconhecidas, mas sabe-se que ela é potencialmente perigosa para mãee filho $(1,2)$ com prevalência de 5,0 a $10,0 \%$ das gestações (3).

As síndromes hipertensivas, juntamente com as hemorragias são as complicações que mais contribuem para a mortalidade materna nos países em desenvolvimento. Na América Latina e Caribe estas mortes representam 25,7 \% e nos países desenvolvidos, $16,1 \%$ (4).

Certamente, a presença de situações que coloquem em risco a vida da gestante e do bebê faz com que estas mulheres sejam mais vulneráveis emocionalmente do que aquelas que não apresentam fatores de risco, principalmente em relação ao estresse, a ansiedade e ao medo da morte do bebê (5-7).

Gestantes com pré-eclâmpsia necessitam de cuidados não apenas em relação ao tratamento biológico, mas também aos aspectos emocionais que envolvem tal doença (7). O diálogo, a capacidade de percepção e a sensibilidade da equipe de saúde são imprescindíveis para o bom andamento da gravidez (8).

Estudos que utilizam abordagem subjetiva para enfocar a narrativa da gestante com pré-eclâmpsia e sua percepção acerca da própria doença tem sido pouco desenvolvidos (9). Os estudos têm abordado mais os aspectos relacionados à percepção de risco na gravidez $(10,11)$, sentimentos relacionados à hospitalização (12) e a satisfação das gestantes com os cuidados no pré-natal e parto (13-15).

Devido à importância da pré-eclâmpsia no cenário mundial se reconhece a necessidade de mais estudos que possam ajudar na diminuição das taxas de morbimortalidade materno-fetal (2). Porém, existem questões relacionadas à dimensão emocional do processo saúde-doença que necessitam ser mais exploradas durante o pré-natal e puerpério. Diante deste fato, o propósito do presente estudo foi compreender como gestantes e puérperas com pré-eclâmpsia, percebiam e sentiam esta doença.

\section{METODOLOGÍA}

A pesquisa foi realizada no ambulatório de pré-natal de alto risco e internamento da Maternidade-Escola Januário Cicco, instituição pública federal de referência para gestação de alto risco, do Estado do Rio Grande do Norte, Brasil, entre fevereiro e junho de 2007. 
A amostra intencional contou com vinte mulheres-dezoito gestantes e duas puérperas-com diagnóstico de pré-eclâmpsia. Os critérios de inclusão das participantes foram: apresentar ou ter apresentado pré-eclâmpsia devidamente diagnosticada (1), saber que estavam apresentando ou que apresentaram pré-eclâmpsia e concordar em participar do estudo. Todas as participantes concordaram e assinaram um termo de consentimento. Na definição do tamanho da amostra foi utilizado o critério de saturação do sentido, o qual é atingido quando as respostas se tornam semelhantes e não há mais situações novas ou divergentes (16).

Entrevista semi-estruturada e observação foram técnicas escolhidas para a obtenção dos dados. A entrevista permite a compreensão aprofundada dos valores, crenças e experiências dos participantes (16) e a observação ajuda na percepção e apreensão de comportamentos individuais e linguagem não-verbal, melhor do que por meio da fala ou da escrita (17). As entrevistas foram baseadas em duas questões norteadoras: "como você acha que a pré-eclâmpsia apareceu na sua vida?" e "como você se sentiu sabendo que tinha pré-eclâmpsia?". As entrevistas foram gravadas em fita cassete e transcritas como editor de texto. A observação foi realizada ao longo de todo o período de coleta dos dados e foi utilizado como instrumento, o diário de campo. Procurou-se observar os momentos de interação das participantes com os profissionais de saúde, como intuito de perceber a dimensão emocional presente nestes contatos, além da maneira como a doença era abordada pelos profissionais e pacientes. Para tal, observou-se o momento das consultas, a sala de monitoramento das gestantes, as reuniões de grupo de gestantes, bem como o horário de visita dos familiares.

Quanto à análise dos dados optou-se por uma perspectiva interpretativa baseada na hermenêutica de Gadamer (18) e auxiliada por autores como Phillips (19) e Fleming (20), que procuraram adaptar esta perspectiva para interpretação dos fenômenos relativos à saúde e doença. Embora Gadamer não tenha desenvolvido propriamente um método para análise e interpretação de dados, sua filosofia tem ajudado pesquisadores qualitativos no desenvolvimento de uma compreensão mais aprofundada dos textos produzidos em suas pesquisas (20). A partir da leitura exaustiva das entrevistas e das anotações do diário de campo, que primeiramente se fez de forma superficial para depois se tornar aprofundada (21) elaborou-se categorias temáticas de acordo com os objetivos da pesquisa, para em seguida construir as categorias empíricas correspondentes a cada tema e que emergiram das entrevistas. No presente estudo, trabalhou-se uma categoria temática experiência da doença - e a partir dele foram construídas as seguintes categorias empíricas: concepções sobre a pré-eclâmpsia, surgimento da pré-eclâmpsia e suas causas e sentimentos em relação à pré-eclâmpsia. 
Segundo Geanellos (21), o sentido escolhido para representar o texto depende de quem o interpreta, de sua orientação acerca do fenômeno, de suas précompreensões. Assim, toda interpretação é uma aproximação, portanto, buscar uma interpretação única e correta para um fenômeno seria negar sua pluralidade. $\mathrm{Na}$ análise final, o leitor é quem decide se aceita, modifica ou rejeita a construção do pesquisador.

A presente pesquisa foi aprovada pelo Comitê de Ética em Pesquisa da Universidade Federal do Rio Grande do Norte.

\section{RESULTADOS}

As participantes tinham em média 26 anos (26,1 $\pm 6,3$ anos), baixo nível de instrução e baixos rendimentos. Metade delas era multípara e solteira, porém viviam em união estável com o pai da criança.

Dentro da categoria temática Experiência da doença, as três categorias empíricas identificadas estão apresentadas nesta seção.

Concepções sobre a pré-eclâmpsia. Para as gestantes e puérperas as concepções sobre a pré-eclâmpsia foram identificadas como: sinais/sintomas e percepções de risco.

Em relação aos sinais/sintomas, as gestantes e puérperas identificaram o que sentiamou percebiam como: pressão alta, "inchaço" dor de cabeça, tontura, vômitos, falta de ar, visão "de estrelinhas" (referência aos escotomas), dor no peito e desmaios. Em alguns momentos, o "passar mal" presente nas falas das gestantes surgiu de forma bastante subjetiva.

\footnotetext{
"Ela (a pré-eclâmpsia) apareceu quando eu vim descobrir eu já tava com quase oito meses, aí meu menino, uns dias que completou oito meses, aí meu menino, eu...deu em mim uma agonia, uma vontade de... de... desesperar, aquele 'fogo' [...]"
}

Também foi possível perceber gestantes que não sabiam o que significava a doença pela qual estavam passando, como mostra o depoimento a seguir:

"[...] até então eu tava com a pressão super alta, no entanto eu não sei não, eu não consigo muito deduzir esta palavra 'pré-ecrâmpsi', porque eu não sei se a mulher fica tremendo em cima duma cama, se a mulher sente alguma coisa, assim, eu não sei [...].

Nas percepções relacionadas ao risco, três aspectos foram marcantes: a morte, as consequiências da doença e o desconhecimento sobre o que poderia acontecer. 
Com relação aos dois primeiros, estas mulheres se manifestaram acerca do risco de morte do bebê, delas próprias e do risco do bebê nascer com algum problema, pois elas percebiam a estreita ligação entre a mãe e o bebê durante a vida intra-uterina. Não se tratava apenas de agravo físico, pois problemas emocionais que a mãe viesse a sofrer na gravidez, como estresse e raiva também teriam repercussão na saúde do bebê.

"Assim, penso assim, pode morrer, né? um negócio de repente, uma coisa que você tá com a pressão muito agoniada e aí como é que fica? O menino dentro da gente pode morrer [...]"

"[...] porque eu acho que o bebê é muito ligado à mãe, pois se eu choro, ou tenho raiva, alguma coisa assim, eu sinto ele mexer, eu acho que isso mexe com ele também, né [...]?

Durante as observações das consultas, algumas mulheres se mostraram ansiosas e angustiadas com a saúde e o bebê, como pode ser visto em duas situações. Na primeira, a gestante estava bastante ansiosa para falar, porém era constantemente interrompida pelas perguntas de rotina do profissional. Na segunda, a gestante descrevia para a profissional várias queixas ao mesmo tempo e mostrava uma série de exames já realizados. Mesmo se mostrando receptiva, a profissional não conseguiu tranqüilizá-la nem satisfazer sua visível ansiedade, pois a maioria das questões postas por ela ficou sem solução.

Surgimento da pré-eclâmpsia. A maneira com que as gestantes e puérperas se expressaram em relação ao surgimento da enfermidade revelou o quanto foram envolvidas de forma inesperada por esta doença: "surgiu do nada", " apareceu de última hora", "foi assim de uma hora para outra..." demonstrando uma ocorrência súbita e um sentimento de surpresa, de algo imprevisível.

Segundo as participantes, a pré-eclâmpsia surgiu devido à hereditariedade, fatores emocionais ou psicológicos, presença de pressão alta e falta de cuidados com alimentação.

A percepção de que as chances de ter pré-eclâmpsia poderiam aumentar quando um parente próximo já tivesse apresentado a doença tornou o surgimento da mesma um evento natural, como algo determinado a acontecer, do qual não se poderia escapar.

"Eu acho que a doença apareceu através da minha família, porque minha mãe já teve préeclâmpsia da primeira gravidez dela passou muito mal, minha irmã já teve começo também, na primeira gravidez e eu tive na primeira gravidez e também tô tendo agora, também..." 
Raiva, preocupação e estresse foram citados como problemas emocionais e psicológicos causadores da doença.

"Por uma raiva que eu tive, começou na dor de cabeça e findou nisso, né?, na pré-eclâmpsia [...] dor de cabeça, dor de cabeça e quando cheguei aqui era pressão alta foi, encaminharam pra urgência [...]"

Nesta categoria houve destaque para problemas no relacionamento com o marido ou companheiro. Em suas falas, as gestantes e puérperas utilizavam frases como: "aborrecimento como o marido", "desavenças no meu casamento". O depoimento que se segue expõe a histórica submissão da mulher ao homem que muitas vezes se traduz em violência física:

"Eu sofri assim na época da minha gravidez, assim, é...tive muitos problemas com relação ao meu parceiro, ele me bateu algumas vezes, né? então, assim, ali começou a alterar meu sistema, eu vivia muito nervosa...e acho que isso acarretou, a questão do parceiro, na época, da questão dele me agredir, eu ficar sempre nervosa, com medo e apreensiva..."

A pressão alta foi destacada em praticamente todas as narrativas, como se este fosse o único risco a que as gestantes estavam expostas. No internamento, as mulheres sabiam que tinham pressão alta, porém metade delas não sabia que apresentava pré-eclâmpsia.

"Não sei bem dizer como foi que apareceu, sei que foi a partir do quarto mês de minha gestação eu comecei a ficar inchada e não tinha nada de pressão alta. Aí foi a partir do sexto ou sétimo mês foi que começou a pressão alta, foi [...]"

"Não sei, talvez pela pressão alta, eu nunca tive, agora nessa gravidez, começou a pressão alta... aí ....apareceu [...]"

Segundo as narrativas das mulheres, a pré-eclâmpsia também poderia surgir devido à falta de cuidados com a alimentação verbalizada como: "excesso de sal", "excesso de gordura", "muita fritura", "comida salgada", "excesso de massa", "excesso de comida".

"Por causa do sal. [...] gosto de comida um pouco salgada. É e também por causa do peso, elas dizem que é também por eu tô fora do peso, aí provoca... Eu engordei na primeira gestação... engordei... comecei com $100 \mathrm{~kg}$ e cheguei a $125 \mathrm{~kg}$ e meio. Quando eu tive, eu fiquei com $119 \mathrm{~kg} . .$. foi quando eu peguei a minha segunda gestação (risos) e agora estou com 127 kg."

"Eu acho que era assim porque eu não me cuidava, assim, é... tipo alimentação, eu comia assim as coisas muito mais forte... muita gordura, muito salgado, essas coisas assim, eu acho que veio através disso aí [...]." 
Algumas participantes não conseguiram uma explicação para o surgimento da doença, pois utilizaram expressões como "não sei explicar...", "não tenho a menor idéia...", "não sei bem dizer como foi que apareceu....". Mesmo não obtendo explicação no pré-natal, uma gestante tentava compreender e construir a história de sua doença, como forma de entender o que estava se passando com ela.

"Na verdade, que eu não tenho até hoje conhecimento, né? do que é pré-eclâmpsia e eu não sei se isso é...é...vem... é...é hereditário ou acontece por acaso, não sei, não tenho ainda...quero até realmente ter um estudo mais aprofundado desse assunto."

Sentimentos em relação à pré-eclâmpsia. $\mathrm{O}$ medo em relação à doença foi o sentimento que predominou nas narrativas. Medo que variou de intensidade e forma dependendo se havia ou não experiência anterior de pré-eclâmpsia. Quando a mulher estava vivenciando a doença pela primeira vez sem apresentar manifestações de gravidade, este sentimento estava ligado à preocupação e angústia em relação ao que ela não conhecia. Já aquelas que manifestaram a doença, com complicações graves, em gestação anterior ou mesmo na atual, o medo surgiu como angústia, trauma, desespero e choro. $\mathrm{O}$ medo de morrer, de perder o bebê ou o medo que a doença surgisse na hora do parto emergiu nos relatos destas mulheres.

"Vixe!, eu fiquei desesperada, pra mim, parecia que o mundo tinha acabado e eu só vivia chorando, desesperada, que é assim, eu pensei assim, não, eu vou morrer, porque é como eu já tinha tido da primeira, né? e tinha sido muito... eu tinha passado um mês e 15 dias internada, então eu, quando eu imaginei que tava grávida, pra mim ali, o mundo tinha se acabado!"

"Ah! Fico triste, né? muito triste... não... assim, o medo da perda do bebê, tenho medo é...também é um risco pouco pra mãe ...tá sofrendo os dois."

"Ah! Tô com um pouco de medo, com o que possa acontecer na hora do parto não sei, de acontecer alguma coisa comigo, com a criança".

Mais uma vez, o medo apareceu como consequiência do desconhecimento sobre a doença, fato que gerou dúvidas sobre o que poderia acontecer e se fez presente em falas como: "coisa nova que estava acontecendo comigo e eu não sabia como lidar" ou "por causa da idade, não tinha assim orientação, não tinha nada...".

\section{DISCUSSÃO}

O modo como se deve compreender e tratar uma doença é influenciado pela cultura local. A narrativa do doente demonstra o quanto os significados sociais e culturais configuram sua experiência e identidade enquanto uma pessoa doente $(22,23)$. Além disso, a narrativa dá voz ao sofrimento de uma forma que ultrapassa o domínio da medicina, vista sob a perspectiva do modelo biomédico (24). 
Nem sempre os sintomas de uma doença aparecem como algo concreto e conhecido pela medicina, pois a percepção da doença está ligada a vários sentidos e muitos deles são ambíguos (22). Isto porque a doença não é apenas a observação de uma disfunção, ela é também o sentimento de angústia pelo desconhecido, a vulnerabilidade em relação às sequielas permanentes e muitas vezes, o temor pela proximidade da morte (28). No presente estudo, o risco da morte, das consequiências da doença e o temor pelo desconhecido estavam presentes nas narrativas das mulheres. Estudo realizado com gestantes de alto risco também demonstrou que elas perceberam riscos relacionados à morte e doença do bebê e à morte da mãe (10). A percepção acerca dos sintomas físicos parece se tornar maior nas gestantes com evolução desfavorável da doença quando comparadas com as que se mantêm estáveis em seu quadro (30).

No tocante ao desconhecimento sobre o que poderia ocorrer devido à presença de pré-eclâmpsia percebeu-se que mesmo as gestantes atendidas no ambulatório se apresentando visivelmente ansiosas com o desenrolar de sua enfermidade, não encontravam espaço para conversar especialmente sobre questões ligadas à dimensão emocional da doença. Provavelmente se os profissionais focassem o atendimento nas queixas relatadas pelas pacientes esquecendo um pouco a anamnese tradicional, as gestantes e puérperas saíssem mais tranquiilas e satisfeitas. Infelizmente, os profissionais de saúde acham que sabem o que os pacientes necessitam e esquecem de escutá-los $(25,26)$. Neste estudo, os profissionais não adotaram uma abordagem que facilitasse o diálogo e a maioria das mulheres acabou por se adaptar a este modelo de consulta e não houve oportunidade para que elas conversassem sobre suas dúvidas e ansiedades. O conflito cultural presente na relação entre profissional de saúde e paciente (23) e a barreira social existente no modelo tradicional de assistência à saúde, que reflete a posição marginalizada da mulher na sociedade (15), principalmente em países em desenvolvimento, provavelmente contribuíram para esta ausência de diálogo. Nãoé fácil para mulheres de baixa renda e baixa instrução questionarem a falta de diálogo e a falta de participação no tratamento, em um serviço de saúde organizado de forma a ver e tratar a doença apenas mediante os sinais e sintomas biológicos (22).

Mesmo ainda não sendo conhecidas as causas que podem levar ao aparecimento da pré-eclâmpsia (1), as gestantes e puérperas buscavam causas que pudessem explicar o porquê do surgimento da pré-eclâmpsia em suas vidas. Dentre os fatores emocionais citados pelas participantes, destacaramse os problemas relacionados aos companheiros, principalmente quando deixaram transparecer ofenômeno da violência doméstica. Infelizmente, a violência contra a mulher se faz presente em todas as classes sociais, etnias, religiões e culturas e representa uma das mais antigas e ina- 
antigas e inaceitáveis expressões da violência de gênero. Tanto em países desenvolvidos quanto em desenvolvimento, milhares de mulheres já sofreram, por parte do companheiro, algum tipo de violência física e ou sexual (27). Provavelmente, muitas outras mulheres e não apenas a do presente estudo passaram por este problema e não encontraram espaço no serviço de saúde para falar sobre esta dramática situação e nem para receber apoio emocional ou mesmo ajuda prática.

O grande destaque dado à pressão alta, pelas gestantes, puérperas e até pelos profissionais colocou em segundo plano ou mesmo desconsiderou outros sintomas também importantes, além de ter dificultado a compreensão da doença em sintonia com aspectos sociais, culturais, emocionais e não apenas biológicos. Percebeu-se que os profissionais, nas raras tentativas de explicar a pré-eclâmpsia para as gestantes, acabaram banalizando a doença e supervalorizando o sintoma mais comum - a hipertensão - além de demonstrarem uma visão reducionista do problema contribuindo para que a gestante também só enxergasse a doença desta forma e não valorizasse outras situações que poderiam ter relação com o agravamento da mesma.

O medo, como principal sentimento percebido neste estudo acabou preenchendo o espaço que deveria ser de sentimentos positivos como alegria e realização pela chegada do bebê. Sousa e colaboradores (9) também observaram que as gestantes manifestavam medo de morrer ou de perder o bebê. Junto com este medo também estava o desconhecimento das participantes em relação à enfermidade. $\mathrm{O}$ desconhecimento tinha relação direta com a falta de diálogo entre profissionais de saúde e paciente, já discutida neste estudo e também por outros autores $(13,15)$, que não ocorre apenas no pré-natal, mas na maioria das consultas de saúde onde os pacientes não são estimulados a falar sobre sua doença, a narrar sua história e expressar seus sentimentos em relação ao fato de estarem doentes (22).

Na medicina ocidental tradicional, o foco do tratamento é o corpo e a doença é vista unicamente como sinais e sintomas $(22,23)$, provavelmente gestantes e puérperas não estão encontrando apoio suficiente para se expressarem acerca de questões ligadas ao estado emocional e isto faz com que o sofrimento seja ainda maior.

O diálogo na saúde não é uma tarefa fácil e exige uma mudança de atitude principalmente do profissional, pois nenhuma pessoa que se veja e se sinta apenas como "um caso do ponto de vista médico" pode realmente ser tratada e nenhum profissional pode ajudar um ser humano a superar sua enfermidade se esse profissional empregar apenas o conhecimento técnico, cartesiano, tão rotineiro de 
sua especialidade e esquecer que a doença está impregnada por sentimentos e experiências muitas vezes difíceis de esquecer e superar (29).

As participantes do estudo demonstraram surpresa e choque com o diagnóstico de pré-eclâmpsia, além de manifestarem medo e ansiedade em relação à doença e suas consequiências para a própria saúde e a do bebê. A percepção da gravidade da pré-eclâmpsia somada a pouca informação e apoio recebidos durante o prénatal e internamento hospitalar agravaram ainda mais este medo e dificultaram a compreensão mais ampla das dimensões biológicas, sociais e emocionais da enfermidade.

Por se tratar de um estudo qualitativo, seus achados não podem ser generalizados para todas as gestantes com pré-eclâmpsia, porém apontam para a necessidade de um novo olhar para a assistência à gestação de alto risco, em especial a préeclâmpsia, no que diz respeito à escuta, acolhida e orientação. Neste sentido, se faz necessário que os profissionais incluam o diálogo em suas consultas, com o intuito de apoiar emocionalmente as pacientes e orientá-las sobre a doença e o tratamento escolhido

Agradecimentos. Ao Programa de Pós-Graduaçãoem Ciências da Saúde/UFRN, Natal, Rio Grande do Norte, Brasil; À Coordenação de Aperfeiçoamento de Pessoal de Nível Superior (CAPES)/Programa de Qualificação Institucional(PQI)/Brasil

\section{REFERÊNCIAS}

1. National High Blood Pressure Education Program. Report of the National High Blood Pressure Education Program Working Group on High Blood Pressure in Pregnancy. Am. J. Obstet. Gynecol. 2000; 183 Suppl 1: 1-22.

2. Sibai B, Dekker G, Kupferminc M. Preeclampsia. Lancet 2005; 365: 785-99.

3. Beckmann CRB, Ling FW, Herbert WNP, Laube DW, Smith RP, Barzansky BM. Hypertension in pregnancy. In: Obstetrics and gynecology. Williamns \& Williamns; 1998; 237-46.

4. Khan KS, Wojdyla D, Say L, Gulmezoglu AM, Van Look PFA. WHO analysis of causes of maternal death: a systematic review. Lancet. 2006; 367: 1066-74.

5. Richter MS, Parkes C, Chaw-Kant J. Listening to the voices of hospitalized high-risk antepartum patients. J. Obstet. Gynecol. Neonatal Nurs. 2007; 36: 313-318.

6. Blackford KA, Richardson H, Grieve S. Prenatal education for mothers with disabilities. J. Adv. Nurs. 2000; 32(4): 898-904.

7. Peters RM, Flack JM. Hypertensive Disorders of Pregnancy. J. Obstet. Gynecol. Neonatal Nurs. 2004; 33(2):209-20.

8. Ministério da Saúde do Brasil. Secretaria de Atenção à Saúde. Departamento de Ações Programáticas Estratégicas. Área Técnica de Saúde da Mulher. Pré-natal e puerpério: atenção qualificada e humanizada. Manual Técnico. 2005; Brasil, Brasília. [internet] Disponível em: http:// bvsms.gov.br/bvs/publicações/caderno5_saude_mulher.pdf Consultado em nov 2006. 
9. Sousa NL, Araújo ACPF, Azevedo GD, Jerônimo SLB, Barbosa LM, Sousa NML. Percepção matema com o nascimento prematuro e vivência da gravidez com pré-eclâmpsia. Rev. Saude Publica 2007; 41(5): 704-10.

10. Gupton A, Heaman M, Cheung LW. Complicated and uncomplicated pregnancies:women's perception of risk. J. Obstet. Gynecol. Neonatal Nurs. 2001; 30(2): 192-201.

11. Heaman M, Gupton A.Gregory D. Factors influencing pregnant women's perceptions of risk. MCN: Am. J. Matern. Child Nursing 2004; 29(2):111-16.

12. Leichtentritt RD, Blumenthal N, Elyassi A, Rotmensch S. High-risk pregnancy and hospitalization: the women's voices. Health Soc. Work 2005; 30(1): 39-47.

13. Harrison MJ., Kusher KE, Benzies K, Rempel G, Kimak C. Women's satisfaction with their involvement in health care decisions during a high risk pregnancy. Birth 2003; 30(2): 109-15.

14. Hildingsson I, Radestad I. Swedish women's satisfaction with medical and emotional aspects of antenatal care. J. Adv. Nurs. 2005; 52(3): 239-49.

15.Sword W. Prenatal care use among women of low income: a matter of «taking care of self». Qual. Health Res. 2003; 13(3): 319-13.

16.Gaskell G Entrevistas individuais e grupais. In: Bauer MW, Gaskell G. Pesquisa qualitativa com texto, imagem e som. 4 ed. Petrópolis: Vozes; 2005; 64-89.

17.Victora CG, Knauth DR, Hassen MNA. Técnicas de pesquisa. In: Pesquisa qualitativa em saúde - uma introdução em saúde. 1 ed. Porto Alegre; 2000; $61-78$.

18.Gadamer HG.Verdade e método. Traços de uma filosofia hermenêutica. 8 ed. Petrópolis: Vozes. São Paulo: Editora Universitária São Francisco; 2007.

19. Phillips B. Nursing care and understanding the experiences of others: a Gadamerian perspective. J. Adv. Nurs. 2007; 14(1): 89-94.

20. Fleming V, Gaidys U, Robb Y. Hermeneutic research in nursing: developing a Gadamerian-based research method. Nursing Inquiry 2003;10: 113-120.

21. Geanellos R. Exploring Ricouer's hermeneutic theory of interpretation as a method of analysing research texts. Nursing Inquiry 2002; 7:112-19.

22. Kleinman A. The Illness narratives: suffering, heading and the human condition. New York: Basic Books; 1988.

23. Sakalys JA. The political role of illness narratives. J. Adv. Nurs. 2000; 31(6):1469-75.

24. Hydén LC. Ilness and narrative. Sociology of Health \& Ilness. 1997; 19(1): 48-69.

25. Blackford KA, Richardson H, Grieve S. prenatal education for mothers with disabilities. J. Adv. Nurs. 2000; 32(4), 898-904.

26. Sheppard VB, Zambrana RE, O'Malley A. Provinding health to low-income women: a matter of trust. Fam. Pract 2004; 21: 484-491.

27. García-Moreno C, Jansen HAFM, Ellsberg M, Helse L, Watts C. WHO multi-country study on women's health and domestic violence against woman. World Health Organization [internet] Disponível em: http://www.who.int/gender/violence/who_multicountry_study/en/index.html. Consultado em fev 2008.

28. Kottow AR, Kottow MH. The disease-subject as a subject of literature. Philosophy, Ethics, and Humanities in Medicine. 2007; 2(10) [internet] Disponível em:http://www.peh-med.com/ content/2/1/10. Consultado em abr 2008.

29. Gadamer HG O caráter oculto da saúde. Petrópolis: Vozes; 2006.

30. Black KD. Stress, symptoms, self-monitoring confidence, well-being, and social support in the progression of preeclampsia/gestational hypertension. J. Obstet. Gynecol. Neonatal Nurs. 2007; 36: 419-29. 